\title{
Ergebnisse der Behandlung der Rhizarthrose im Frühstadium mittels der Distraktionsarthroplastik nach Bufalini und Perugia
}

\author{
Results after Distraction Arthroplasty According to Bufalini and Perugia in Early \\ Stages of Trapeziometacarpal Osteoarthritis
}

\author{
Autoren \\ L. Fatzer ${ }^{1}$, E. Soleman ${ }^{2}$, T. Sanchez ${ }^{1}$ \\ Institute \\ ${ }^{1}$ Handchirurgie, Orthopädische Klinik, Kantonsspital Olten, Schweiz \\ ${ }^{2}$ Plastische-, Rekonstruktive-, Ästhetische- und Handchirurgie, Universitätsspital Basel, Schweiz
}

\begin{abstract}
Schlüsselwörter
- Rhizarthrose

- Distraktionsarthroplastik nach Bufalini und Perugia

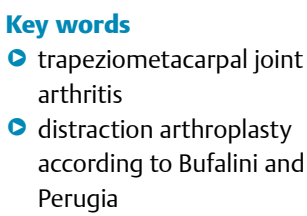

- distraction arthroplasty according to Bufalini and Perugia

eingereicht 14.1.2014 akzeptiert $\quad 3.1 .2015$

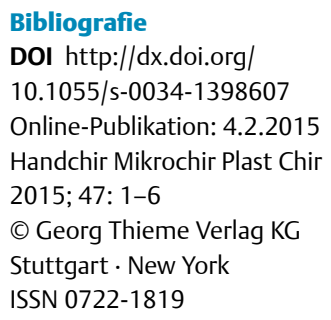

Korrespondenzadresse

\section{Dr. Lukas Fatzer}

Handchirurgie

Kantonsspital Olten

Baselerstrasse 150

4600 Olten

Schweiz

Tel.: + 41/623/114 875

Fax: $+41 / 623 / 114449$

fatzer@hotmail.com

\section{Zusammenfassung}

$\nabla$

Ziel: Die vorliegende, retrospektive Studie untersucht die Behandlungsergebnisse der von Bufalini und Perugia zur Behandlung der Daumensattelgelenksarthrose im Frühstadium beschriebenen Distraktionsarthroplastik. Hierbei wird ein freies Palmaris longus-Sehnentransplantat distal am 2. Mittelhandknochen befestigt und quer durch die erste Kommissur und die Basis des Metakarpale I gezogen, sodass eine Distraktion des Sattelgelenkes resultiert.

Patienten und Methoden: Bei 9 Patienten mit persistierenden Beschwerden nach konsequenter konservativer Behandlung einer Rhizarthrose Grad I-II nach Eaton wurden 10 Distraktionsarthroplastiken nach Bufalini und Perugia durchgeführt. Aufgrund erneuter Schmerzen war bei 2 Patienten im Verlauf eine Trapezektomie notwendig. Diese wurden von der Nachuntersuchung ausgeschlossen. Die verbliebenen 7 Patienten mit 8 Distraktionsarthroplastiken wurden in Bezug auf Patientenzufriedenheit, Schmerzen sowie Daumenbeweglichkeit als auch Kraft beim Grob-, Schlüssel- und Spitzgriff nach durchschnittlich 46,5 (29-63) Monaten nachuntersucht. Die Kraft wurde dabei absolut gemessen, aber auch in Relation zur Gegenseite prozentual bestimmt. Zur Erfassung der Beweglichkeit des Daumenstrahles wurde beidseits die kombinierte Flexions-Oppositionsbewegung mittels des Kapandji-Index bestimmt und die Abspreizung des Metakarpale I vom Metakarpale II gemessen. Des Weiteren wurde der DASHScore erfasst und eine Röntgenkontrolluntersuchung vorgenommen.

Ergebnisse: Alle 7 nachuntersuchten Patienten waren mit dem Operationsresultat subjektiv zufrieden. Die Patienten erreichten beim Grobgriff $86,1 \%(23,1 \mathrm{~kg} \pm 4,8 \mathrm{~kg})$ des Wertes der Gegenseite, beim Spitzgriff $86,3 \%(4,8 \mathrm{~kg} \pm 0,9 \mathrm{~kg})$ und beim Schlüsselgriff $80,1 \%(5,9 \mathrm{~kg} \pm 1,1 \mathrm{~kg})$.

\section{Abstract}

$\nabla$

Backgound: A distraction arthroplasty of the trapeziometacarpal joint was introduced by Bufalini and Perugia for the treatment of the early stages of carpometacarpal osteoarthritis. Our retrospective study presents the results of this technique. Thereby, a tendon graft anchored to the distal second metacarpal is fixed at the base of the first metacarpal, keeping it in distraction. Material and Methods: 10 distraction arthroplasties were performed in 9 patients with carpometacarpal osteoarthritis stage I and II of the thumb after unsuccessful conservative therapy. In 2 cases, which were excluded from our study, trapeziectomy had to be performed because of persisting pain. Patient satisfaction, pain measurement, range of motion, and tip, key and grip strength were evaluated at a follow-up of 46.5 (29-63) months in the remaining 7 patients (8 operations overall). Strengh measurement was taken in an absolute value and compared to the opposite side. Thumb range of motion was measured with the combined flexion-opposition of the thumb with the Kapandij index and also the angle of abduction of the metacarpal I to metacarpal II. Assessment included a DASH score evaluation and an X-ray control.

Results: All of the 7 evaluated patients were satisfied with the operation results. Compared to the opposite side, patients achieved $80.1 \%$ $(5.9 \mathrm{~kg} \pm 1.1 \mathrm{~kg})$ of key pinch strength, $86.3 \%$ $(4.8 \mathrm{~kg} \pm 0.9 \mathrm{~kg})$ of oppositional tip pinch strength, and $86.1 \%(23.1 \mathrm{~kg} \pm 4.8 \mathrm{~kg})$ of grip strength. In combined flexion and opposition a Kapandij index of 8.5 (94.4\%) compared to 9 on the opposite side was achieved. Thumb radial abduction was $48.2^{\circ} \pm 2.8^{\circ}$, compared to $51.0^{\circ} \pm 2.9^{\circ}$ on the contralateral hand. At follow-up, the mean DASH score was $17.8( \pm 10.0)$. Radiological control showed no progression of carpometacarpal osteoarthritis of the thumb. 
In der kombinierten Flexions-Oppositionsbewegung wurde ein Kapandij-Index von 8,5 (94,4\%) erreicht, auf der Gegenseite ein Index von 9. Die Daumenabduktion in der Hohlhandebene betrug auf der operierten Seite 48,2 ( $\pm 2,8)$ Grad, auf der Gegenseite 51,0 $( \pm 2,9)$ Grad. Der DASH-Score betrug 17,8 $( \pm 10,0)$ Punkte. Radiologisch wurde keine Progression der Rhizarthrose festgestellt.

Schlussfolgerung: Unsere Ergebnisse nach Distraktionsarthroplastik nach Bufalini und Perugia bestätigen die Wirksamkeit der Operation im frühen Stadium der Rhizarthrose. Auch wenn die Versagerquote mit 20\% hoch war, halten wir den Eingriff im Frühstadium der Rhizarthrose für berechtigt, zumal die meisten Patienten über einen längeren Zeitraum von der Operation profitieren und die Option einer späteren Trapezektomie erhalten bleibt.

\section{Einleitung}

$\nabla$

Die Indikation zum operativen Vorgehen bei der Rhizarthrose ist ganz wesentlich von den Schmerzen des Patienten abhängig. Bei der Entscheidungsfindung wie operativ vorgegangen wird, spielt letztlich bei der fortgeschrittenen Rhizarthrose im Stadium III und IV nach Eaton und Littler ( $\bullet$ Tab. 1) die Vorliebe des Operateurs die entscheidende Rolle, wobei zusätzlich vorhandene degenerative Veränderungen des Gelenkes zwischen Kahnbein, großem und kleinem Vieleckbein der Beachtung bedürfen [1]. Zur Anwendung kommen die alleinige Trapezektomie, Resektions-Suspension-Interpositionsplastiken, die Suspension mittels Mini TightRope ${ }^{\circledR}$, Pyrocarbon Implantate, Gelenkprothesen als auch die Arthrodese des trapeziometakarpalen Gelenks [2-8].

Sofern sich noch keine oder nur geringe Veränderungen im Röntgenbild zeigen (Stadium I und II nach Eaton), andererseits nur wenig Schmerzen bestehen, wird in der Regel konservativ therapiert $[9,10]$. Bei Formvariante des Trapeziums mit abgeflachter distaler Gelenkfläche kommen aufrichtende Korrekturosteotomien in diesen frühen Stadien in Betracht [11-13]. Liegt eine Instabilität mit Subluxation des Gelenkes vor, kommt zur Stabilisierung die Bandplastik nach Eaton-Littler zur Anwendung, bei der ein Streifen der Sehne des M. flexor carpi radialis verwendet wird [14]. Allerdings berichteten bereits Eaton und Littler in ihrer Originalpublikation, dass die Ergebnisse abhängig vom Vorhandensein und dem Ausmaß arthrotischer Veränderungen schlechter ausfallen.

2007 beschrieben Bufalini und Perugia [15] für Frühstadien der Rhizarthrose mit und ohne Subluxation eine Distraktionsarthroplastik, bei der ein freies Palmaris longus-Sehnentransplantat distal am Metakarpale II verankert, das freie Ende quer durch die erste Kommissur und dann unter Spannung durch ein Bohrloch in der Basis des Metakarpale I gezogen wird, sodass es zur Distalisierung des Metakarpale I und somit zur Entlastung des Sattelgelenkes kommt. Beeindruckt durch die bestechend einfache und logische Konstruktion begannen wir 2008 die von Bufalini und Perugia beschriebene Operation bei Patienten mit persistie-
Conclusions: The collected data after distraction arthroplasty according to Bufalini and Perugia confirm the efficacy of the technique in the early stage of carpometacarpal osteoarthritis of the thumb. Even though a failure rate of $20 \%$ occurred, in our opinion the operation is justified in the early stages of carpometacarpal osteoarthritis of the thumb as the patients benefit from a pain-free interval, leaving the option for future trapeziectomy.

renden Beschwerden nach konservativer Behandlung einer Rhizarthrose Grad I-II nach Eaton durchzuführen. Ziel dieser Arbeit war eine Re-Evaluation der von uns operierten Patienten, um zu prüfen, ob sich das Verfahren wirklich bewährt und dies auch bei längeren Verläufen.

\section{Patienten und Methode \\ $\nabla$}

\section{Patienten}

Von April 2008 bis November 2010 haben wir 10 Distraktionsarthroplastiken an 9 Patienten durchgeführt. Bei 2 Patienten wurde im weiteren Verlauf nochmals eine Operation notwendig, wobei einmal eine alleinige Trapezektomie und im anderen Fall eine Trapezektomie mit Interpositions-Suspensions-Arthroplastik nach Lundborg [16] erfolgte. Diese beiden Patienten wurden von der Nachuntersuchung ausgeschlossen. Somit konnten 7 Patienten mit 8 operierten Händen nachuntersucht werden. Es handelt sich um 6 Frauen $(87,5 \%)$ und 1 Mann (12,5\%) mit einem mittleren Alter zum Zeitpunkt der Operation von 47,6 Jahre (32-51 Jahren). Eine Frau wurde beidseits operiert. 5-mal war die linke, adominante Hand und 3-mal die rechte, dominante Hand betroffen.

In allen Fällen lag eine Daumensattelgelenksarthrose Grad I-II nach Eaton vor. Bei allen Patienten war längerfristig eine konservative Therapie mit Tragen einer Schiene, Ergotherapie als auch eine Gelenkinfiltration mit Kortison erfolglos durchgeführt worden.

Alle 9 Patienten waren zum Zeitpunkt der Operation berufstätig. Von den beiden Patienten, die im Verlauf einer Trapezektomie bedurften, übte eine Patientin eine die Hände belastende, die andere eine die Hände weniger belastende Tätigkeit aus. 6 der 7 nachuntersuchten Patienten hatten eine die Hände belastende Arbeit. Die beidseits operierte Patientin arbeitet im Detailhandel ohne große Belastung ihrer Hände ( $\bullet$ Tab. 2).

\section{Operationstechnik}

Der Zugang zum Daumensattelgelenk (CMC-1-Gelenk) erfolgt über eine $3 \mathrm{~cm}$ lange Inzision und Darstellung des Gelenkes

Tab. 1 Eaton und Littler Klassifikation.
Stadium I:
Normale Gelenkkonturen, erweiterter Gelenkspalt (Erguss).
Stadium II:
Geringe Gelenkspaltverschmälerung, evtl. Osteophyten kleiner als 2 mm.
Stadium III:
Sklerotische und zystische Veränderungen im subchondralen Knochen, Osteophyten größer als 2 mm, Zerstörung des Daumensattelgelenkes. Normale trapezioskaphoidale Gelenkfläche.
Stadium IV:
Zusätzlich zum Verschleiß der trapeziometakarpalen Gelenkfläche finden sich degenerative Veränderungen im Bereich der trapezioskaphoidalen Gelenkfläche. 


\begin{tabular}{|rllllll|}
\begin{tabular}{|rlll} 
Patient/ \\
In
\end{tabular} & Alter & Geschlecht & Beruf & $\begin{array}{l}\text { Operierte } \\
\text { Seite }\end{array}$ & $\begin{array}{l}\text { Dominante } \\
\text { Seite }\end{array}$ & $\begin{array}{l}\text { Nachkontrolle } \\
\text { (Monate nach OP) }\end{array}$ \\
\hline 1 & 37 & W & Serviceangestellte & R & R & ausgeschlossen \\
\hline 2 & 57 & W & Büroangestellte & R & R & ausgeschlossen \\
\hline 3 & 48 & W & OP-Pflegerin & L & R & 43 \\
\hline 4 & 48 & W & Serviceangestellte & L & R & 48 \\
\hline 5 & 57 & W & Bäuerin & L & R & 54 \\
\hline 6 & 43 & W & Fabrikmitarbeiterin & R & R & 63 \\
\hline 7 & 56 & W & Serviceangestellte & L & R & 60 \\
\hline 8 & 41 & W & Detailhandel & R & R & 29 \\
\hline 9 & 41 & W & Detailhandel & L & R & 36 \\
\hline 10 & 48 & m & Fabrikmitarbeiter & R & R & 39 \\
\hline
\end{tabular}

Tab. 2 Patientenkollektiv.
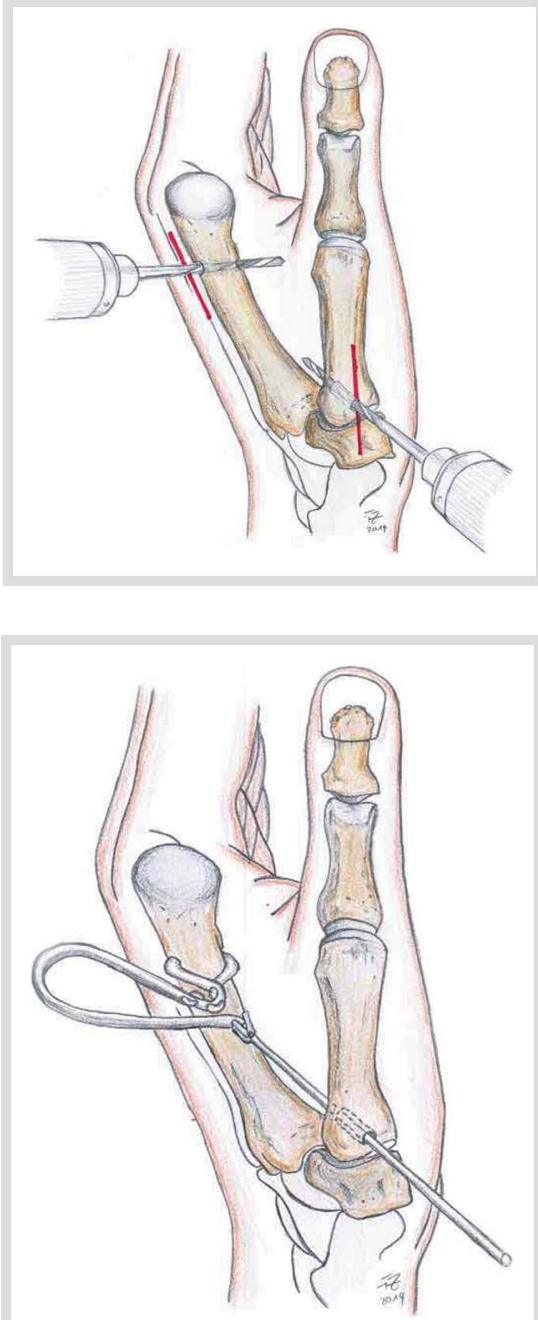

Abb. 1 Anbringen eines Bohrkanal $(2,5 \mathrm{~mm})$ im distalen Anteil des Metakarpale II und an der Basis Metakarpale I. (Quelle: Dr. L. Fatzer).

Abb. 2 Verankerung der Palmaris longus Sehne mittels Pulvertaft-Naht am. Metakarpale II und Durchziehen durch die erste Kommissur und Basis Metakarpale I mittels Arthroskopiezange $(1,9 \mathrm{~mm})$. (Quelle: Dr. L. Fatzer).

mittels Längsarthrotomie zur Inspektion des Gelenkes und gleichzeitig Orientierung des Bohrkanals. Mit einem 2,5 mm Bohrer wird ein schräg ansteigender Bohrkanal von proximal radial nach distal ulnar in Richtung auf den Kopf des Metakarpale II (MC II) angelegt. Über eine zweite kurze Längsinzision wird das distale Drittel des Schaftes des MC II dargestellt und ebenfalls mittels 2,5 mm Bohrer ein Bohrkanal erstellt ( $\bullet$ Abb. 1). Die Sehne des M. palmaris longus (PL) wird über kurze Inzisionen gewonnen. Das freie Sehnentransplantat wird durch das Bohrloch im MC II gezogen und mittels Pulvertaftnaht mit sich vernäht. Durch das Bohrloch im MC I wird eine 1,9 mm Arthroskopiezange durch die erste Kommissur in Richtung auf das Bohrloch im MC II eingebracht ( $\bullet$ Abb. 2) und das freie Ende der

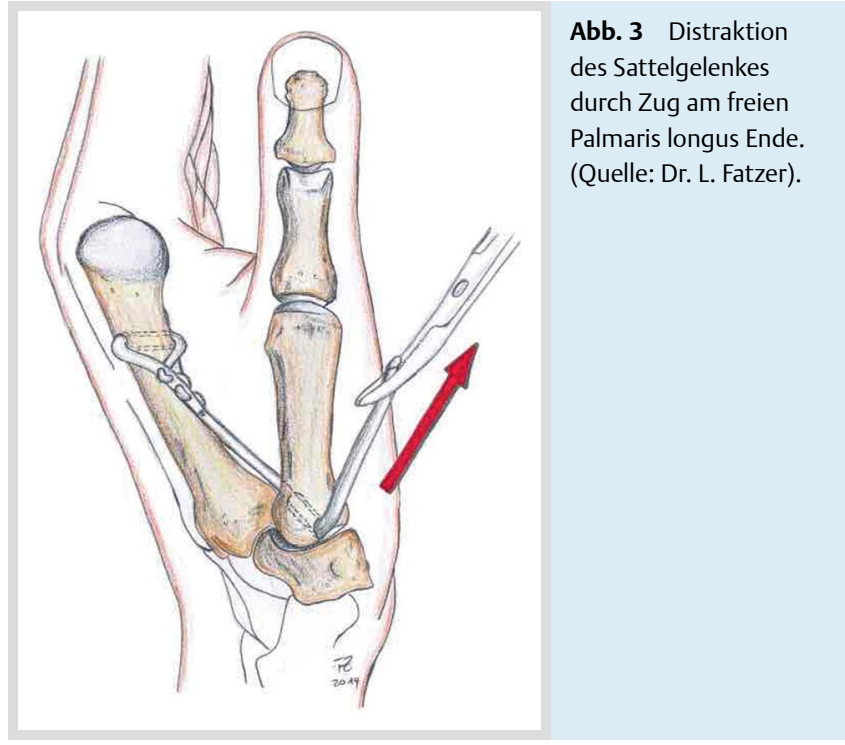

PL-Sehne gefasst und durch den Bohrkanal an der Basis des MC I gezogen. Unter Zug wird die PL-Sehne am Periost des MC I mit PDS 3.0 Z-Nähten fixiert ( $\bullet$ Abb. 3,4). Dadurch wird eine Distraktion und Reposition des Gelenkes erreicht, welche mittels Bildwandlerdurchleuchtung kontrolliert wird. Der Wundverschluss erfolgt in üblicher Weise.

\section{Nachbehandlung}

Direkt postoperativ wird eine Daumenvorderarmschiene angepasst, welche nach Abschwellung der Weichteile eine Woche postoperativ gegen einen geschlossenen Daumengips für weitere 3 Wochen ersetzt wird. 4 Wochen postoperativ wird die Schiene entfernt und mit Bewegungsübungen unter Anleitung der Handtherapeuten für 2 Wochen ohne Belastung und für weitere 2 Wochen mit Belastungsaufbau begonnen. Eine volle Belastung der Hand ist nach 8 Wochen erlaubt. Die Nachkontrollen wurden nach 1, 4 und 8 Wochen nach der Operation durchgeführt.

\section{Klinische und radiologische Untersuchung}

Die Nachuntersuchung erfolgte im Durchschnitt 46,5 (29-63) Monate postoperativ. Alle 7 Patienten wurden klinisch untersucht und detailliert über Beschwerden, Funktion und Zufriedenheit befragt. Die Schmerzintensität wurde mittels einer visuellen Analogskala (VAS, 0 Punkte für keinen Schmerz, 10 Punkte für unerträglichen Schmerz) erfasst. Die Beweglichkeit des Daumensattelgelenkes wurde im Vergleich zur Gegenseite bestimmt. Der Kapandji-Index ( $\bullet$ Abb. 5) [17] wurde dabei zur Quantifizierung der kombinierten Flexion-Opposition verwen- 

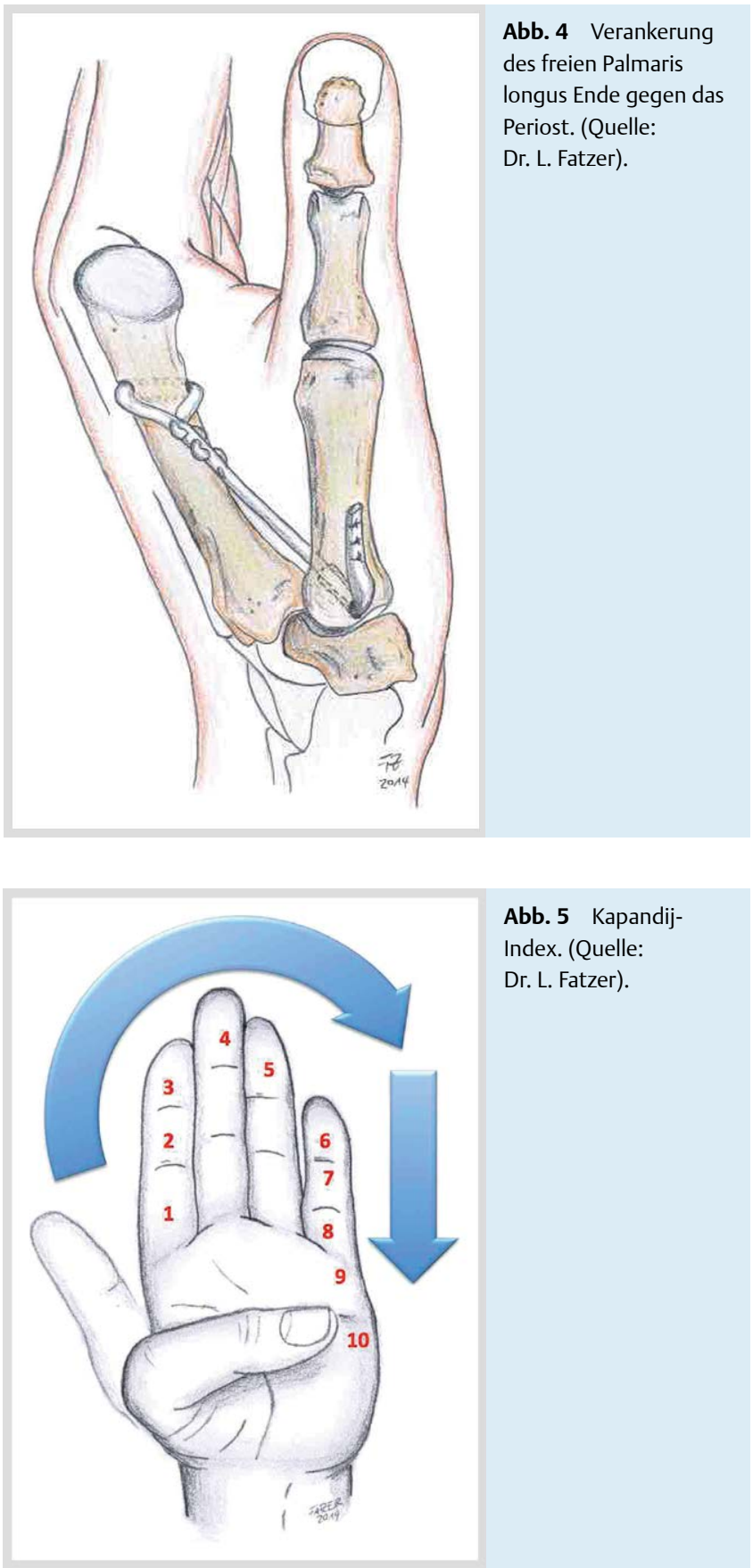

det und die radiale Abduktion des Daumenstrahles in der Hohlhandebene mittels eines Goniometers für kleine Gelenke gemessen. Die Kraftmessungen erfolgten ebenfalls im Seitenvergleich mit dem Jamar-Dynamometer (Baseline Hydraulic Hand Dynamometer, Fabrication Enterprise Intercorporated, Irvington, NY, USA) für den Grobgriff sowie mit einem Kneif-Dynamometer (Baseline Pinch-Gauge, Orthopartner AG, Seon, Schweiz) für den Schlüssel- und Spitzgriff. Dabei wurde für jeden Test aus jeweils 3 Messungen der Mittelwert errechnet. Zusätzlich wurde der DASH-Fragebogen [18] von den Patienten ausgefüllt. Des Weiteren wurde bei der Nachuntersuchung zur Beurteilung des Metakarpale I und II sowie des Sattelgelenkes eine Röntgenuntersuchung mit Aufnahme der Hand in antero-posteriorer und schräger Stellung vorgenommen.

\section{Statistik}

Die Resultate wurden deskriptiv als Mittelwerte und Standardabweichungen sowie Minimum/Maximum dargestellt.

\section{Ergebnisse}

\section{Komplikationen}

Intraoperativ sind keine Komplikationen aufgetreten. Bei einer Patientin kam es postoperativ zu einer Tendovaginitis stenosans de Quérvain, welche unter handtherapeutischen Maßnahmen und einer einmaligen Steroidinfiltration folgenlos abheilte.

\section{Objektive Messresultate}

Die kombinierte Flexions-Oppositions-Bewegung ergab im Mittel einen Kapandji-Index von 8,5 bzw. 94,4\% im Vergleich zur Gegenseite mit einem mittleren Index von 9. Die Daumenabduktion nach radial in der Hohlhandebene war auf der operierten Seite im Durchschnitt bis 48,2 $( \pm 2,8)$ Grad möglich, entsprechend 94,6\% der Gegenseite, wo sie im Durchschnitt 51,0 $( \pm 2,9)$ Grad betrug.

Bei der postoperativen Auswertung der Kraftentwicklung wurde die beidseitig operierte Patientin miteingeschlossen. Die Patienten erreichten im Mittel beim Grobgriff $86,1 \%(23,1 \mathrm{~kg} \pm 4,8 \mathrm{~kg})$ des Wertes der Gegenseite, beim Spitzgriff $86,3 \%(4,8 \mathrm{~kg} \pm 0,9 \mathrm{~kg})$ sowie beim Schlüsselgriff $80,1 \%(5,9 \mathrm{~kg} \pm 1,1 \mathrm{~kg})$.

\section{Zufriedenheit}

Mit dem Operationsergebnis waren in 6 Fällen (75\%) die Patienten „sehr zufrieden“ und in 2 Fällen (25\%) „zufrieden“. Kein Patient war mit dem Resultat „unzufrieden“ bzw. „sehr unzufrieden“. Die Schlüsselfrage nach Wiederholung der Operation bei gleichen Symptomen bejahten alle 7 untersuchten Patienten.

\section{Schmerzen}

Beschwerden wurden insbesondere bei längerer Maximalbelastung im Alltag angegeben. Die Beurteilung der Schmerzen im Alltag auf der VAS ergab einen Mittelwert von 1,6 ( $\pm 1,3)$ Punkten. Im Vergleich mit den Schmerzen vor Operation wurde der aktuelle Zustand in 5 Fällen als ,viel besser“ und in 3 Fällen als ,besser" beurteilt.

Die vor der Operation ausgeübte Arbeit wurde von allen 7 Patienten weitergeführt. Es gab keinen Arbeitsplatzwechsel und keine Reduktion des Arbeitspensums. In einem Fall wurde die präoperativ ausgeübte Freizeitbeschäftigung (Tennis) nach der Operation aufgegeben.

\section{Radiologische Befunde}

Nach einer mittleren Verlaufszeit von 46,5 (29-63) Monaten nach der Operation wurde radiologisch in keinem der 8 nachkontrollierten Fälle eine Progression des Arthrosestadiums festgestellt $(\bullet$ Abb. 6a, b).

\section{DASH}

Die Auswertung des DASH-Fragebogens ergab im allgemeinen Teil bei 8 korrekt ausgefüllten Bögen einen Mittelwert von 17,8 $( \pm 10,0)$ Punkten. Im optionalen Teil „Arbeit“ wurde bei 7 beantworteten Fragekatalogen ein Mittelwert von 10,5 ( $\pm 15,6$ ) Punkten und beim Teil „Freizeit“, der nur 2 ausgefüllt worden war, 0 bzw. 25 Punkten errechnet. 


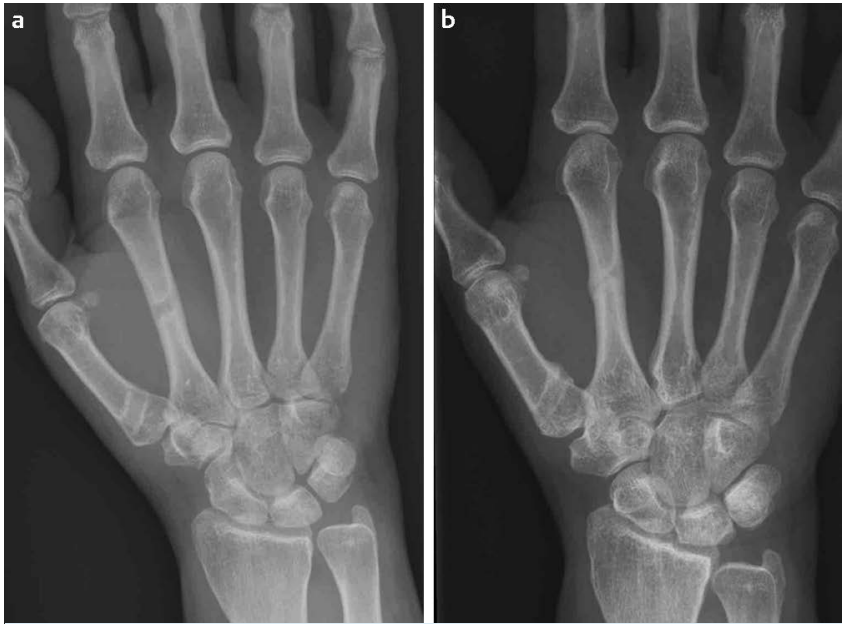

Abb. 6 Röntgenkontrolle einer 43-jährigen Fabrikarbeiterin nach Operation $\mathbf{a}$ und nach 63 Monaten $\mathbf{b}$.

\section{Diskussion}

$\nabla$

Einen Konsensus in der Behandlung der Eaton Stadien I und II der Rhizarthrose gibt es bisher nicht. Dies bestätigt eine 2012 publizierte Online-Befragung von 1156 Kollegen der Amerikanischen Gesellschaft für Handchirurgie [10]. Bei der Frage nach der Behandlung einer 42-jährigen Patientin mit Rhizarthrose im Stadium I-II nach Eaton, welche nach Schienentherapie und Gelenkinfiltration mit Kortison persistierende Beschwerden habe, gaben $46 \%$ der Befragten an, die konservative Therapie weiterzuführen, für ein Trapezium erhaltendes operatives Vorgehen waren 34\%. Davon würden 13\% die Ligamentplastik nach Eaton-Littler [14], 11\% die Osteotomie des Metakarpale nach Tomaino [12] und 10\% eine Arthroskopie mit Débridement des Gelenkes durchführen. Insgesamt $18 \%$ erachteten bereits in diesem frühen Stadium eine Resektions-Interpositions-Arthroplastik für indiziert. Das Ergebnis der Umfrage zeigt einerseits, wie unterschiedlich das Vorgehen im frühen Stadium der Daumensattelgelenksarthrose bei Versagen konservativer Maßnahmen ist, andererseits aber auch, dass die große Mehrheit (80\%) in diesem Stadium trotz persistierender Beschwerden nach konservativer Therapie die Resektion des Trapeziums noch nicht für indiziert hält.

Feine Arthroskopie-Instrumente ermöglichen heute die arthroskopische Behandlung der Rhizarthrose im Frühstadium. Betroffene Patienten profitieren von einem Débridement, Synovektomie und Kapsulorafie [19]. Culp und Rekant erreichten mit dieser Methode bei 22 Patienten und 24 operierten Daumen in $88 \%$ der Fälle exzellente oder gute Resultate bei einer Nachkontrollzeit zwischen 14 Monaten und 4 Jahren nach Operation.

Badia und Khanchandani [20] berichteten über kombinierte Eingriffe mit Arthroskopie und nachfolgender Extensions-Osteotomie in Frühstadium der Rhizarthrose. Dabei wurden bei $43 \mathrm{~Pa}-$ tienten eine Arthroskopie mit gleichzeitiger Synovektomie und je nach Befund Kapsulorafie durchgeführt, gefolgt von einer Extensions-Osteotomie. Sie berichten über zufriedenstellende Ergebnisse in Bezug auf Schmerzfreiheit, Stabilität und Kraft beim Faustschluss.

Verlaufsdaten nach arthroskopischer Behandlung von Frühstadien beschränken sich jedoch bisher auf kleine Fallzahlen und kurze Verlaufszeiten.
Die Stabilisierungsoperation nach Eaton-Littler wird als prophylaktisches Vorgehen bei Instabilität des Daumensattelgelenkes beschrieben, wobei von den ersten 18 von Eaton und Littler behandelten Patienten einige bereits eine Arthrose aufwiesen. Bei der Operation wird ein Streifen der Sehne des M. flexor carpi radialis abgespalten und durch ein distal durch die Basis des Os metacarpale I gebohrtes Loch gezogen, dann unter der Abductor-pollicis-longus-Sehne hindurch und um die Flexor-carpiradialis-Sehne geführt und schließlich am Periost vernäht. Nyszkiewicz und Neumann [21] erzielten mit der Methode bei 7 Patienten überwiegend ein gutes Ergebnis hinsichtlich der Stabilität, der Daumenkraft und der subjektiven Zufriedenheit. Eaton und Littler [14] selbst erzielten bei 18 Patienten 11 sehr gute, 5 gute und 2 schlechte Ergebnisse, wobei die Ergebnisse mit Vorhandensein arthrotischer Veränderungen schlechter ausfielen.

Insgesamt verlor die Methode in den letzten Jahren etwas an Beachtung, nicht zuletzt zu Gunsten der Abduktions-ExtensionsOsteotomie, welche erstmals von Wilson 1973 beschrieben wurde [22]. Diese führt durch die Verlagerung der Belastung des Knorpels von palmar nach dorsal zu einer Beschwerdeabnahme sowie einer Verbesserung der Faustschluss und Schlüsselgriffkraft [23]. Parker fand bei einer mittleren Nachuntersuchungszeit von 9 (6-13) Jahren bei 6 von 8 Patienten nach ExtensionsOsteotomie ein gutes Ergebnis [13]. Tomaino berichteten über eine Serie von 12 Patienten mit einer Nachkontrollzeit von 25 (6-46) Monaten und 11 zufriedene Patienten. Als ungeeignet erachtet Tomaino die Methode bei Patienten mit Subluxationsstellung und fortgeschrittenem Arthrosestadium [24].

Die von uns verwendete, von Bufalini und Perugia 2007 erstmals beschriebene Distraktionsarthroplastik stellt eine weitere Methode für eine Trapezium erhaltende Operation dar, welche die Subluxationsstellung korrigiert [15]. Bei einer mittleren Nachuntersuchungszeit von 18 Monaten waren alle 25 von Bufalini und Perugia operierten Patienten komplett oder fast komplett beschwerdefrei. Messwerte der Beweglichkeit als auch der Kraft nach der Operation wurden leider nicht berichtet. Die Operationstechnik wurde im Vergleich zur Trapezektomie als schneller, einfacher und weniger invasiv bezeichnet.

Unbefriedigt durch die erfolglose konservative Therapie und mit dem Wunsch das Trapezium insbesondere bei jungen Patientinnen mit frühen Rhizarthrosestadien zu belassen, begann der Seniorautor (T.S.) 2008 mit der von Bufalini beschriebenen Distraktionsarthroplastik. In 2 der ersten 10 Fälle kam es nach wenigen Monaten zu erneuten Beschwerden. Bei erneut nachweisbarer Subluxationsstellung musste eine Insuffizienz der Distraktionsarthroplastik angenommen werden. Bei intraoperativ suffizienter Distraktionsarthroplastik erfolgte in einem Fall eine alleinige Trapezektomie. Im anderen Fall wurde bei intraoperativ fraglich suffizienter Aufhängung zusätzlich zur Trapezektomie eine Suspension-Interpositions-Arthroplastik nach Lundborg durchgeführt [16]. Da es sich um 2 der ersten Fälle dieser Serie handelte, wurde im weiteren Verlauf ein besonderes Augenmerk auf eine stabile Verankerung des freien Palmaris longus-Transplantates gegen das Periost an der Basis des Metakarpale I gelegt. Für die Nachkontrolle wurden diese 2 Fälle aufgrund des geänderten Situs nach Trapezektomie ausgeschlossen. In unserer retrospektiven Studie konnte die Kraftentwicklung nur mit der Gegenseite verglichen werden. Dieser Vergleich hat eine limitierte Aussagekraft, da weder die Händigkeit noch allfällige Erkrankungen der Gegenseite berücksichtigt werden. Präoperative Messwerte waren leider nicht systematisch erfasst worden, sodass retrospektiv ein Vergleich möglich gewesen 
wäre. Ebenfalls der Vergleich mit anderen Studien hat eine begrenzte Aussagekraft aufgrund der kleinen Fallzahlen und des gemischten Patientenkollektiv. Die gemessenen Mittelwerte (Grobgriff 23,1 kg, Spitzgriff 4,8 kg Schlüsselgriff 5,9 kg) liegen über den Werten nach Extensions-Osteotomie sowohl von Parker (Grobgriff $19 \mathrm{~kg}$, Spitzgriff $3 \mathrm{~kg}$ und Schlüsselgriff $5 \mathrm{~kg}$ ) [13] als auch Tomaino (Grobgriff $8,5 \mathrm{~kg}$, Spitzgriff 3,0 kg) [24]. Auch die von Nyszkiewicz und Neumann postoperativ nach EatonLittler-Bandplastik bei 7 Patienten nach 12 (5-36) Monate gemessene Kraft beim Spitzgriff und beim Grobgriff war mit $3,5 \mathrm{~kg}(34,3 \mathrm{~N})$ bzw. 11,5kg $(113,1 \mathrm{~N})$ deutlich niedriger als unsere Ergebnisse [21]. Mit 17,8 Punkten im DASH-Score wiesen unsere Patienten eine deutlich geringere Einschränkung im Alltag im Vergleich zu den Patienten Nyszkiewicz und Neumann mit 22,92 Punkten auf. Als zusätzlichen Wert bestimmten wir die Daumenabduktion in der Handebene, da man aufgrund des Sehnenverlaufs vom MC I durch das MC II bei der Distraktionsarthroplastik nach Bufalini und Perugia den Verdacht hegen muss, dass dies die Abduktion eingeschränkt. Die Messung ergab mit 48,2 (94,6\%) auf der operierten Seite im Gegensatz zur Gegenseite mit 51,0 $0^{\circ}$ nur eine geringe Einschränkung. Bei der Gesamtbeurteilung des Operationsergebnisses bejahten die nachuntersuchten Patienten die Schlüsselfrage nach Zufriedenheit mit dem Operationsergebnis und alle würden die Operation bei denselben Beschwerden wiederholen. Daraus schließen wir, dass trotz gewisser Restbeschwerden (VAS 1,6 Punkte) eine hohe Patientenzufriedenheit erreicht werden kann.

\section{Schlussfolgerung}

$\nabla$

Ein Konsens punkto operativer Therapie der Frühstadien der Rhizarthrose ist noch nicht gefunden und wohl auch nicht zu erwarten. Unsere Ergebnisse nach Distraktionsarthroplastik bestätigen die Wirksamkeit der Operation auch über einen längeren Zeitverlauf. Nach der Durchführung einer Distraktionsarthroplastik wird die Option einer späteren Trapezektomie nicht ausgeschlossen.

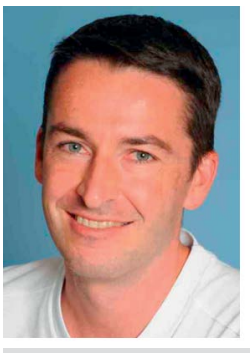

Lukas Fatzer

Geb. am 1.2.1974 in Bern, Schweiz. 1994-2001 Medizinstudium an der Universität Bern. 2001 Dissertation Universität Bern. 2002 Assistenzarzt Chirurgie Regionalspital Langenthal. 2003 Assistenzarzt Pädiatrie Kantonsspital Münsterlingen. 2004-2006 Assistenzarzt Chirurgie Regionalspital Langenthal, Kantonsspital Aarau und Olten. 2007 Oberarzt i.V. Chirurgie Kantonsspital Olten. 2009 Facharzttitel FMH für Chirurgie. 2010-2012 Oberarzt Handchirurgie Kantonsspital Olten und Graubünden. 2012 Assistenzarzt und von 2013-2014 Oberarzt Handchirurgie Kantonsspital Aarau. Seit Juli 2014 Leitender Arzt Handchirurgie Kantonsspital Olten.

\section{Interessenkonflikt: Nein}

\section{Literatur}

1 Eaton RG, Glickel SZ, Littler JW. Tendon interposition arthroplasty for degenerative arthritis of the trapeziometacarpal joint of the thumb. J Hand Surg 1985; 10: 645-654

2 Schibli-Beer S, Mark G, Canova M. Ergebnisse nach alleiniger Trapezektomie und Kapselraffung bei der Behandlung der Rhizarthrose. Handchir Mikrochir Plast Chir 2008; 40: 169-174

3 Szalay G, Scheufens T, Alt V et al. Die operative Versorgung der therapierefraktären Rhizarthrose mittels Trapezektomie und Suspension des ersten Strahls mittels Mini TightRope ${ }^{\circledR}$. Handchir Mikrochir Plast Chir 2014; 46: 179-185

4 Szalay G, Meyer C, Kraus $R$ et al. Die operative Versorgung der Rhizarthrose mittels Pyrocarbonspacer als Trapeziumersatz. Handchir Mikrochir Plast Chir 2009; 41: 300-305

5 Barrera-Ochoa S, Vidal-Tarrason N, Correa-Vázquez E et al. Pyrocarbon interposition (PyroDisk) implant for trapeziometacarpal osteoarthritis: Minimum 5-year follow-up. J Hand Surg Am 2014; 39: 2150-2160

6 Mariconda M, Russo S, Smeraglia $F$ et al. Partial trapeziectomy and pyrocarbon interpositional arthroplasty for trapeziometacarpal joint osteoarthritis: results after minimum 2 years of follow-up. J Hand Surg Eur 2014; 39: 604-610

7 Vermeulen GM, Slijper H, Feitz R et al. Surgical management of primary thumb carpometacarpal osteoarthritis: a systematic review. J Hand Surg Am 2011; 36: 157-169

8 Chug M, Williams N, Benn D et al. Outcome of uncemented trapeziometacarpal prosthesis for treatment of thumb carpometacarpal joint arthritis. Indian J Orthop 2014; 48: 394-398

9 Yao J, Park MJ. Early treatment of degenerative arthritis of the thumb carpometacarpal joint. Hand Clin 2008; 24: 251-261

10 Moriatis WJ, Delaronde S. Current trends in nonoperative and operative treatment of trapeziometacarpal osteoarthritis: A survey of US hand surgeons. J Hand Surg 2012; 37: 77-82

11 Wilson JN. Basal osteotomy of the first metacarpal in the treatment of arthritis of the carpmetacarpal joint of the thumb. Br J Surg 1973; 60: $854-858$

12 Tomaino MM. Treatment of Eaton stage I trapeziometacarpal disease with thumb metacarpal extension osteotomy. J Hand Surg 2000; 25: $1100-1106$

13 Parker WL, Linscheid RL, Amadio PC. Long-term outcomes of first metacarpal extension osteotomy in the treatment of carpal-metacarpal osteoarthritis. J Hand Surg Am 2008; 33: 1737-1743

14 Eaton $R G$, Littler $W$. Ligament reconstruction for the painful thumb carpometacarpal joint. J Bone Joint Surg Am 1973; 55: 1655-1666

15 Bufalini C, Perugia D. Distraction arthroplasty of the trapeziometacarpal joint. J Hand Surg 2007; 32: 1443-1446

16 Sigfusson R, Lundborg G. Abductor pollicis longus tendon arthroplasty for treatment of arthrosis in the first carpometacarpal joint. Scand J Plast Reconstr Surg Hand Surg 1991; 25: 73-77

17 Kapandij A. Bioméchanique des articulations trapéziométacarpienne e scapho-trapézienne. In: Saffar P. (ed.). La rhizarthrose. Paris, Expansion Scientifique Francaise. 1990; 30: 67-70

18 Germann G, Harth W, Wind $G$ et al. Standardisierung und Validierung der deutschen Version 2.0 des „Disability of Arm, Shoulder, Hand“ (DASH)-Fragebogens zur Outcome-Messung an der oberen Extremität. Unfallchirurg 2003; 106: 13-19

19 Culp RW, Rekant MS. The role of arthroscopy in evaluating and treating trapeziometacarpal disease. Hand Clin 2001; 17: 315-319

20 Badia A, Khanchandani P. Treatment of early basal joint arthritis using a combined arthroscopic debridement and metacarpal osteotomy. Techniques in Hand and Upper Extremity Surgery 2007; 11: 168-173

21 Nyszkiewicz R, Neumann R. Erfahrungen mit der Eaton-Littler-Plastik zur Therapie der Bandinstabilität am Daumensattelgelenk. Handchir Mikrochir Plast Chir 2001; 33: 424-430

22 Wilson JN. Arthroplasty of the trapezio-metacarpal joint. Plast Reconstr Surg 1972; 49: 143-148

23 Cooney WP III, Lucca MJ, Chao EY et al. The kinesiology of the thumb trapeziometacarpal joint. J Bone Joint Surg Am 1981; 63: 1371-1381

24 Tomaino MM. Basal metacarpal osteotomy for osteoarthritis of thumb. J Hand Surg 2011; 36: 1076-1079 